\title{
Erratum
}

\section{Erratum to: Comparative Value of Cholinesterase Inhibitors and Memantine in Persons with Moderate-to-Severe Alzheimer's Disease in the United States: A Cost-Effectiveness Analysis}

Ismaeel Yunusa, Saud Alsahali, Amey Rane and Tewodros Eguale

Pre-press 19 October 2021

[Journal of Alzheimer's Disease Reports, 5(1) (2021), 705-713, 10.3233/ADR-210307]

https://content.iospress.com/articles/journal-of-alzheimers-disease-reports/adr210307

When this article was first published the third author's name was inadvertently misspelled as "Amey Ranes". This has been corrected to "Amey Rane" in the revised online version of the article (DOI: 10.3233/ADR-210307).

Therefore, the correct updated list of authors and their affiliation is:

Ismaeel Yunusa ${ }^{\mathrm{a}}$, Saud Alsahali ${ }^{\mathrm{b}}$, Amey Rane $^{\mathrm{c}}$ and Tewodros Eguale

${ }^{a}$ Center for Outcomes Research and Evaluation, Department of Clinical Pharmacy and Outcomes Sciences, University of South Carolina College of Pharmacy, Columbia, SC, USA

${ }^{\mathrm{b}}$ Department of Pharmacy Practice, Unaizah College of Pharmacy, Qassim University, Qassim, Saudi Arabia ${ }^{\mathrm{c}}$ MCPHS University, Boston, MA, USA 